\title{
THE FUNDAMENTAL REGION FOR A FUCHSIAN GROUP*
}

BY L. R. FORD

1. Introduction. The present paper is an attempt to lay the groundwork of the theory of Fuchsian groups by basing the treatment on concepts of a very simple sort. The fundamental region to which we are led is not new. It is given by the Fricke-Klein methodt under certain circumstances and is identical with that given by Hutchinson in an important paper. However, we make use neither of non-euclidean geometry nor of quadratic forms, and we are able to derive the major results of the theory of Fuchsian groups in an unexpectedly simple manner.

2. The Group. Given a group of linear transformations with an invariant circle or straight line $K^{\prime}$, the interior of $K^{\prime}$ (or the half-plane on one side of $K^{\prime}$ ) being transformed into itself by each transformation of the group. We shall assume that there exists a point $A$, not on $K^{\prime}$, such that there are no points congruent to $A$ in a sufficiently small neighborhood of $A$.

Let $G$ be a linear transformation carrying $K^{\prime}$ into the unit circle $K$ with center at the origin and carrying $A$ to the origin. Let $S$ be any transformation of the group; then the set of transformations

$$
T=G S G^{-1}
$$

is a group with $K$ as principal circle. $\S$ Configurations

* Presented to the Society, September 11, 1925.

$\dagger$ Fricke-Klein, Vorlesungen über die Theorie der automorphen Funktionen, vol. I, Chap. II.

¥J. I. Hutchinson, $A$ method for constructing the fundamental region of a discontinuous group of linear transformations, TransACTIONS OF THIS Societr, vol. 8 (1907), pp. 261-269.

$\S$ We use this order to mean the transformation $G^{-1}$, followed by $S$, followed by $G$. That is, writing $z^{\prime}=S(z), z^{\prime}=G(z)$, etc., as the combining transformations, the new transformation is $z^{\prime}=T(z)=G\left\{S\left[G^{-1}(z)\right]\right\}$. 
which are congruent by transformations of the new group are carried by $G^{-1}$ into configurations which are congruent by transformations of the original group. It will suffice, then, to find a fundamental region for the new group.

The condition that

$$
z^{\prime}=T(z)=\frac{a z+b}{c z+d}
$$

leave the unit circle unchanged is that $b=\bar{c}, d=\bar{a}$, where bars indicate conjugate imaginaries. Then

$$
T=\frac{a z+\bar{c}}{c z+\bar{a}} .
$$

Since the origin, 0 , must transform into an interior point, and $T(0)=\bar{c} / \bar{a}$, we must have $|c|<|a|$. Hence the determinant $a \bar{a}-c \bar{c}$, which is real, must be positive. We shall insert such a positive factor in numerator and denominator that

$$
a \bar{a}-c \bar{c}=1 .
$$

There is no point congruent to 0 in a suitably small neighborhood of 0 . In particular, 0 is not a fixed point for any transformation; hence $c \neq 0$, unless $T$ be the identical transformation.

3. Two Locus Problems. Excluding the identical transformation, we shall solve the following two locus problems for the transformation $T$.

I. Find the locus of a point in the neighborhood of which lengths and areas are unchanged in magnitude.

Infinitesimal lengths are multiplied by $\left|T^{\prime}(z)\right|$ and infinitesimal areas are multiplied by $\left|T^{\prime}(z)\right|^{2}$. Since

$$
T^{\prime}(z)=\frac{1}{(c z+\bar{a})^{2}},
$$

we have as the required locus the circle

or

$$
C: \quad \begin{aligned}
|c z+\bar{a}| & =1, \\
|z+\bar{a} / c| & =1 /|c| .
\end{aligned}
$$


This circle, which will be called the $C$-circle of the transformation $T$, has its center at the point $-\bar{a} / c$; its radius is $1 /|c|$.

Writing the relation $a \bar{a}-\bar{c} \bar{c}=1$ in the form

$$
1+\frac{1}{|c|^{2}}=\left|\frac{\vec{a}}{c}\right|^{2}
$$

we see that the sum of the squares of the radii of $K$ and $C$ is equal to the square of the distance between their centers. Hence $C$ is orthogonal to $K$. It follows that 0 is outside $C$.

If $z$ is within $C$ then

$$
|z+\bar{u} / c|<1 /|c|, \quad|c z+\bar{a}|<1, \quad\left|T^{\prime}(z)\right|>1,
$$

and lengths and areas in the neighborhood of $z$ are increased in magnitude when transformed by $T$. Similarly, if $z$ is outside $C$ lengths and areas are decreased in magnitude when transformed by $T$.

II. Find the locus of a point whose distance from 0 is unchanged.

The required locus is

or

$$
|z|=\left|\frac{a z+\bar{c}}{c z+\bar{a}}\right|
$$

or

$$
\left|c z^{2}+\bar{a} z\right|=|a z+\bar{c}|,
$$

$$
\left(c z^{2}+\bar{a} z\right)\left(\bar{c} \bar{z}^{2}+a \bar{z}\right)=(a z+\bar{c})(\bar{a} \bar{z}+c) .
$$

On expanding and making use of the relation $c \bar{c}=a \bar{\imath}-1$ this factors into

$$
[z \bar{z}-1][(c z+\bar{a})(\bar{c} \bar{z}+a)-1]=0,
$$

whence

$$
|z|=1, \text { or }|c z+\bar{a}|=1 .
$$

The complete locus, then, consists of the two circles $C$ and $K$.

If $z$ is inside both $C$ and $K$ or outside both, the first member of (a) is greater than 0 , and we find, on retracing our steps, that

$$
|z|>\left|\frac{a z+\bar{c}}{c z+\bar{a}}\right| \text {. }
$$


[Nov.-Dec.,

The transform of $z$ is nearer 0 than $z$ is. Similarly, if $z$ is inside one circle and outside the other the transform is farther from 0 than $z$ is.

4. Geometric Interpretation of $T$. The inverse of $T$,

has the $C$-circle

$$
T^{-1}=\frac{-\bar{a} z+\bar{c}}{c z-a},
$$

$$
C^{\prime}: \quad|z-a / c|=1 /|c| .
$$

It is clear that $T$ carries $C$ into $C^{\prime}$. For, $T$ carries $C$ into a circle $C_{0}$ without alteration of lengths; then $T^{-1}$ transforms $C_{0}$ without alteration of lengths; whence $C_{0}$ coincides with $C^{\prime}$.

The region exterior to both $C$ and $C^{\prime}$ is transformed by $T$ into the interior of $C^{\prime}$ and by $T^{-1}$ into the interior of $C$. Let $z$ be a point of the region, and let $z$ be carried by $T$ and $T^{-1}$ into $z^{\prime}$ and $z^{\prime \prime}$ respectively. In both cases there is diminution of lengths and areas near $z$ since $z$ is exterior to both $C$-circles. Then the inverses, $T^{-1}$ and $T$, carry $z^{\prime}$ and $z^{\prime \prime}$ respectively back to $z$ with increase of lengths and areas. Hence $z^{\prime}$ is in $C^{\prime}$ and $z^{\prime \prime}$ is in $C$.

Let $A, B$ and $A^{\prime}, B^{\prime}$ be the intersections of $C$ and $C^{\prime}$ with $K$, the points being so designated that motion around $C$ from $A$ to $B$ inside $K$ is counter-clockwise about $C$, and motion from $B^{\prime}$ to $A^{\prime}$ around $C^{\prime}$ in $K$ is counter-clockwise about $C^{\prime}$. Now, on applying $T$, the interior arc $A B$ of $C$ is carried without alteration of length into either $A^{\prime} B^{\prime}$ or $B^{\prime} A^{\prime}$. The latter is impossible, for it is equivalent to a suitable rotation with 0 as fixed point, which is contrary to hypothesis. Hence $A$ is transformed into $A^{\prime}$ and $B$ into $B^{\prime}$.

We can now give simple geometric interpretations of $T$. Let $L$ be the perpendicular bisector of the line joining the centers of $C$ and $C^{\prime}$. $\quad L$ passes through 0 . (In the special case that $C$ and $C^{\prime}$ coincide let $L$ be the line joining 0 to the center of $C$.) Either of the following pairs of inversions transforms the points of $C$ exactly as $T$ does and hence is identical with it: 
(1) A reflection in $L$ followed by an inversion in $C^{\prime}$;

(2) An inversion in $C$ followed by a reflection in $L$.

We can show from these inversions that $T$ is hyperbolic, elliptic, or parabolic according as $C$ and $C^{\prime}$ are exterior to one another, intersect, or are tangent. The fixed points of the transformation are easily found geometrically.

5. The Arrangement of the C-Circles. We shall show first that there is an upper bound of the radii of the $C$-circles defined by the transformations of the group. There exists, by hypothesis, a circle of radius $\varepsilon<1$ with 0 as center having no point congruent to 0 in its interior. We have then for any transformation of the group

$$
|T(0)|=\left|\frac{\bar{c}}{\bar{a}}\right| \geqq \varepsilon, \text { whence }\left|-\frac{\bar{a}}{c}\right| \leqq \frac{1}{\varepsilon} ;
$$

that is, the distance from 0 to the center of $C$ is not greater than $1 / \varepsilon$. Since 0 is outside $C$ it follows that the radius of $C$ is less than $1 / \varepsilon$.

Let

$$
T_{1}=\frac{\alpha z+\bar{\gamma}}{\gamma z+\bar{\alpha}}
$$

be a second transformation of the group. Designate by $C_{1}, C_{1}^{\prime}$ the $C$-circles of $T_{1}$ and $T_{1}^{-1}$. Thus $C_{1}$ is $|z+\bar{\alpha} / \gamma|=1 /|\gamma|$. Let us now make the transformation

$$
T T_{1}^{-1}=\frac{(-a \bar{\alpha}+\bar{c} \gamma) z+a \bar{\gamma}-\bar{c} \alpha}{(-c \bar{\alpha}+\bar{a} \gamma) z+c \bar{\gamma}-\bar{a} \alpha},
$$

which, since $T_{1} \neq T$, is not the identical transformation. Designating the radii of $C, C_{1}$, and the $C$-circle of $T T_{1}^{-1}$ by $r, r_{1}, r_{2}$, respectively, we have

$$
r_{2}=\frac{1}{|-c \bar{a}+\bar{a} \gamma|}=\frac{1}{|c \gamma| \cdot\left|-\frac{\bar{\alpha}}{\gamma}-\left(-\frac{\bar{a}}{c}\right)\right|}=\frac{r r_{1}}{d},
$$

where $d$ is the distance between the centers of $C$ and $C_{1}$. Since $r_{2}<1 / \varepsilon$ we have

$$
d=\frac{r r_{1}}{r_{2}}>\varepsilon r r_{1}
$$


Consider now all $C$-circles whose radii equal or exceed some positive number $k$. The distance between the centers of any two of the circles satisfies the inequality

$$
d>\varepsilon k^{2} \text {. }
$$

Since the centers lie in the finite region bounded by $K$ and by the circle of radius $1 / \varepsilon$ with center at 0 it follows that the number of such circles is finite.

It follows from the result just found that any closed region lying entirely within $K$, for example a circle with 0 as center and radius less than 1 , is exterior to all but a finite number of $C$-circles.

Another consequence is that the transformations of the group are denumerable, since the radii of the corresponding $C$-circles are denumerable.

6. The Fundamental region. Let $R$ be the region within $K$ which lies outside all $C$-circles formed for the transformations of the group. The region is connected, since any of its points can be joined to 0 by a straight line segment which does not cross the boundary. We shall show that $R$ is a fundamental region for the group; that is, (1) that no two interior points of $R$ are congruent, and (2) that no region adjacent to $R$ and lying in $K$ can be added to $R$ without the inclusion of points congruent to points of $R$.

The proof of the first property is immediate. The transform of any interior point of $R$ by any transformation of the group, the identical transformation excepted, lies within some $C$-circle and hence is exterior to $R$.

To establish the second property we shall show first that if $P$, a point of $C$, the $C$-circle of some transformation $T$ of the group, lies on the boundary of $R$, then $P^{\prime}$, the tranisform of $P$ by $T$, also lies on the boundary of $R$. $P^{\prime}$ lies on $C^{\prime}$, the $C$-circle of $T^{-1}$.

Suppose $P^{\prime}$ does not lie on the boundary of $R$. Then $P^{\prime}$ lies within the $C$-circle of some transformation $T_{1}$ of the group. Consider the transformation $T_{1} T$. By the trans- 
formation $T$ lengths in the neighborhood of $P$ are carried without alteration of magnitude into the neighborhood of $P^{\prime}$. By $T_{1}$ lengths in the neighborhood of $P^{\prime}$ are magnified. Hence $T_{1} T$ magnifies lengths in the neighborhood of $P$; consequently $P$ is within the $C$-circle of $T_{1} T$. This is contrary to hypothesis; hence $P^{\prime}$ is on the boundary of $R$.

It follows from the preceding that if an arc $a b$ of $C$ forms part of the boundary of $R$ the congruent arc $a^{\prime} b^{\prime}$ of $C^{\prime}$ is a part of the boundary. The transformation $T^{-1}$ carries $R$ into a region abutting $R$ along $a b$. Any region adjacent to $R$ abuts along some $C$-circle and contains points congruent by a suitable transformation to points of $R$. The second property is thus established.

The following properties of the region $R$ are consequences of the preceding analysis:

1. $R$ is bounded by arcs of circles orthogonal to $K$. The number of bounding ares in a circle $|z|=r<1$ is finite.

2. The bounding arcs are arranged in congruent pairs. Two congruent arcs of the boundary are equal in length, and congruent points thereof are equidistant from the center of $K$.

3 . The vertices of a cycle (congruent vertices) lie on a circle concentric with $K$, since all are equidistant from the center of $K$. If the vertices of a cycle lie within $K$ their number is finite.

4. $R$ is the fundamental region of maximum area. For, a different fundamental region must contain points congruent to all points of $R$, and a shift of any part of $R$ to a congruent position effects a diminution of area.

We shall now prove that $R$ and the regions congruent to it fill up without overlapping the whole interior of $K$.

Suppose $R_{i}$ and $R_{j}$, the transforms of $R$ by $T_{i}$ and $T_{j}$, overlap. Let $z_{1}, z_{2}, z_{3}$ be three points common to $R_{i}$ and $R_{j}$. These are the transforms by $T_{i}$ of three points $z_{1}^{\prime}, z_{2}^{\prime}, z_{3}^{\prime}$ of $R$, and the transforms by $T_{j}$ of three points $z_{1}^{\prime \prime}, z_{2}^{\prime \prime}, z_{3}^{\prime \prime}$ of $R$. If $z_{1}^{\prime}=z_{1}^{\prime \prime}, z_{2}^{\prime}=z_{2}^{\prime \prime}, z_{3}^{\prime}=z_{3}^{\prime \prime}$ then $T_{i}$ and $T_{j}$ are the same transformation, since they transform three points in the 
same way, and $R_{i}$ and $R_{j}$ coincide. Otherwise the points of one pair, say $z_{1}^{\prime}$ and $z_{1}^{\prime \prime}$, are unequal. Being both congruent to $z_{1}$ they are congruent, which is impossible.

Let $K_{r}$ be the circle $|z|=r<1$. Let $a_{1} b_{1}, a_{1}^{\prime} b_{1}^{\prime}, \ldots, a_{n} b_{n}, a_{n}^{\prime} b_{n}^{\prime}$ be the sides of $R$ lying wholly or in part within $K_{r}$; and let $T_{i}, i=1,2, \ldots, n$, be the transformation carrying $a_{i} b_{i}$ into $a_{i}^{\prime} b_{i}^{\prime}$. The transforms of all remaining sides of $R$ are exterior to $K_{r}$, since the distance from 0 of any point on such a side is not decreased by any transformation of the group.

By applying $T_{1}, \ldots, T_{n}$ and their inverses we get regions congruent to $R$ abutting on $R$ along the sides $a_{1} b_{1}, \ldots, a_{n}^{\prime} b_{n}^{\prime}$. The sides of the new regions which lie in $K_{r}$ are congruent to the sides just mentioned. By combinations of $T_{1}, \ldots, T_{n}$ and their inverses we can adjoin further regions along sides of these new regions, provided the sides lie in $K_{r}$; and the process can be continued as long as there are any free sides in $K_{r}$.

This process will end in a finite number of steps; for, each transformation carries $R$ into the interior of a particular $C$-circle, and there is but a finite number of $C$-circles intersecting $K_{r}$. Hence $K_{r}$ is covered by a finite number of regions. Since $r$ may be chosen as near 1 as we like, it follows that the whole interior of $K$ is covered.

We note from the preceding that all transformations of the group are formed by combinations of the transformations connecting congruent sides of $R$. These are therefore called generating transformations of the group. A further interesting fact is that a transformation which carries $R$ into a region lying wholly or in part in a circle $K_{r}$ concentric with $K$ is a combination of those generating transformations only whose $C$-circles intersect $K_{r}$.

7. An Important Special Case. The sides of $R$ may be finite or infinite in number. There are certain groups in which a fundamental region not extending to the principal circle is known to exist; for example, some of the groups 
arising in connection with the uniformization problem.* For this case we have the following proposition.

If there exists a fundamental region $F$ lying within $K_{r}(|z|=r<1)$, then $R$ lies within $K_{r}$, and the number of sides and of generating transformations is finite.

$R$ and a finite number of its transforms, $R_{1}, \ldots, R_{m}$, will cover $F$ completely. Carry the portion of $F$ lying in each $R_{i}$ into $R$ by means of the transformation which carries $R_{i}$ into $R$. The totality of these transforms of parts of $F$, together with the portion of $F$ originally in $R$, fill up $R$ completely. If this were not so we could construct a region $D$ adjacent to one of these transformed regions and containing no points congruent to points of $F$. On carrying $D$ back to the boundary of $F$ we should have a region abutting on $F$ and containing no points congruent to points of $F$, which is contrary to hypothesis.

Finally $R$ is in $K_{r}$, for on transforming the parts of $F$ into $R$ the distance of no point from 0 is increased.

Since only a finite number of $C$-circles intersect $K_{r}$, it follows that $R$ has a finite number of sides and that the number of generating transformations is finite.

The Rice Institute

* See Osgood, Lehrbuch der Funktionentheorie, vol. I, 2 d ed., pp. 721-25. 